\title{
Considerations for Phage Therapy Against Mycobacterium abscessus
}

\author{
Abrar Senhaji-Kacha, Jaime Esteban* and Meritxell Garcia-Quintanilla \\ Department of Clinical Microbiology, IIS-Fundación Jiménez Díaz, Madrid, Spain
}

There is a global increasing number of Mycobacterium abscessus infections, especially pulmonary infections. Reduced therapeutic options exist against this opportunistic pathogen due to its high intrinsic and acquired levels of antibiotic resistance. Phage therapy is a promising afresh therapy, which uses viruses to lyse bacteria responsible for the infection. Bacteriophages have been recently administered under compassionate use to a 15-year-old patient infected with $M$. abscessus in combination with antibiotics with

OPEN ACCESS

Edited by:

Wladimir Sougakoff, INSERM U1135 Centre d'Immunologie et de Maladies Infectieuses, France

Reviewed by:

Regis Tournebize, INSERM U1135 Centre d'Immunologie et de Maladies

Infectieuses, France

Jean Louis Herrmann,

Université de Versailles SaintQuentin-en-Yvelines, France

*Correspondence: Jaime Esteban jestebanmoreno@gmail.com

Specialty section:

This article was submitted to Antimicrobials, Resistance and Chemotherapy,

a section of the journal

Frontiers in Microbiology

Received: 23 September 2020 Accepted: 24 December 2020 Published: 18 January 2021

Citation:

Senhaji-Kacha A, Esteban J and

Garcia-Quintanilla M (2021) Considerations for Phage Therapy Against Mycobacterium abscessus.

Front. Microbiol. 11:609017. doi: 10.3389/fmicb.2020.609017 excellent results. This mini review highlights different recommendations for future phage administrations such as where to look for new phages, the use of cocktail of mycobacteriophages to broaden phage specificity and to tackle resistance and phage insensitivity due to temperate phages present in bacterial genomes, the combined use of phages and antibiotics to obtain a synergistic effect, the liposomal administration to reach a prolonged effect, intracellular delivery and protection against neutralizing antibodies, and the convenience of using this strategy in patients suffering from cystic fibrosis (CF) since phages are believed to promote immunomodulatory actions and eliminate biofilms.

Keywords: Mycobacterium abscessus, non-tuberculous mycobacteria, phage therapy, alternative therapy, antibiotic resistance, bacteriophage

\section{INTRODUCTION}

There is a global concern about the increasing number of multidrug resistant bacteria. The Centers for Disease Control (CDC) and the World Health Organization (WHO) have declared antibiotic resistance a threat to global health. Despite the increasing number of resistant bacteria, there is a decreasing effort in manufacturing new antibiotics from pharmaceutical companies. From 1983 to 1987, 16 new antibiotics were approved by the Food and Drug Administration (FDA) in the United States, however, between 2010 and 2016 only six new antibiotics were authorized (Luepke et al., 2017).

Importantly, Mycobacterium abscessus is the most challenging species of mycobacteria due to its intrinsic and acquired bacterial resistance. In the last decade, M. abscessus infections have increased including pulmonary infections, systemic and/or disseminated infections, and skin and soft-tissue infections (Johansen et al., 2020). In pulmonary infection, $M$. abscessus causes chronic respiratory diseases in patients suffering from underlying lung diseases, such as cystic fibrosis, bronchiectasis, and chronic obstructive pulmonary disease. This increment is due to a growing number of patients with immunosuppression and increased life expectancy, as well as other factors that predispose to these infections (Chalmers et al., 2018). 
Mycobacterium abscessus present a phenotypic heterogeneity based on the presence (smooth) or absence (rough) of glycopeptidolipids (GPLs) extracellularly, rough derivatives exhibiting higher levels of virulence than smooth variants (Catherinot et al., 2007; García-Coca et al., 2019).

Phage therapy is a promising afresh therapy, which uses viruses infecting bacteria to deal with infections. In 2014, the United States National Institute of Infectious Diseases included phage therapy as one of seven strategies to tackle antibiotic resistance (Reardon, 2014). A cocktail of three bacteriophages has been recently administered under compassionate use to a 15 -year-old patient infected with $M$. abscessus in combination with antibiotics. The excellent results obtained show that this approach can help to cure infections against this particular pathogen.

\section{CURRENT TREATMENT AGAINST MYCOBACTERIUM ABSCESSUS}

Mycobacterium abscessus is a rapidly growing non-tuberculous mycobacteria (NTM) and an opportunistic pathogen. Treatments last from months to years and are associated to potential risky antibiotic toxicity and a high number of failures. This species is composed by three subspecies: M. abscessus subsp. abscessus, subsp. bolletii and subsp. massiliense (Tortoli et al., 2016) although its taxonomy remains under debate.

Current recommendations to treat $M$. abscessus depend on subspecies. While subspecies massiliense is sensitive to macrolides, subspecies abscessus and bolletii are usually resistant due to the presence of an inducible methylase encoded by the erm(41) gene (Nash et al., 2009).

According to the recommendations found in the official ATS/ ERS/ESCMID/IDSA Clinical practice guideline (Daley et al., 2020), a susceptibility-based treatment for macrolides and amikacin is suggested over empiric therapy in patients with $M$. abscessus pulmonary disease. A multidrug regimen consisting of three or more active drugs is recommended. Importantly, a macrolidecontaining treatment is recommended not only in patients without inducible or mutational resistance, but also under macrolide resistance if macrolides are used for immunomodulatory reasons. The duration of the therapy will depend on each infection. Additional recommendations can be found in the British Thoracic Society guidelines (Haworth et al., 2017).

Novel therapeutic options, including old drugs, iron chelators, or antimicrobial peptides show different degrees of activity against M. abscessus (Bento et al., 2020; Meir and Barkan, 2020; Muñoz-Egea et al., 2020). In this review, we will focus on phage therapy as an alternative therapy against M. abscessus multiresistance.

\section{PHAGE THERAPY AS ALTERNATIVE THERAPY}

Bacteriophages are viruses that infect bacteria and after infection can be lysogenic or lytic. Therapeutic phage use was discovered a century ago, however, after the development of antibiotics, only Eastern Europe continued using this alternative under compassionate use. The current reintroduction in Western countries is caused by the increasing number of multidrugresistant bacteria and the scarce number of novel antibiotics. The first patient treated with phage therapy in the US suffered from a systemic infection caused by a multidrug-resistant Acinetobacter baumannii and completely recovery was achieved (Schooley et al., 2017).

Recent reviews have shown the efficacy and the state of the art of phage therapy research applied to humans, typically, phages are delivered by intravenous or topical route more than once until bacterial count decrease and clinical improvement is achieved. In 2019, McCallin et al. (2019) highlighted different cases of phage therapy under compassionate use. Patey et al. (2018) reviewed all local compassionate use of phages combined with antibiotics at the Villeneuve Saint Georges Hospital (France), describing complete recovery in 12 out of 15 cases, two cases of improvement without eradication, and one eradication with a secondary pathogen emerging. Recent reviews about clinical trials describe results of phage therapy to treat chronic otitis, infected burn wounds, diarrhea, diabetic foot ulcers, venous leg ulcers, urinary tract infections, or gastrointestinal disorders caused by Staphylococcus aureus, A. baumannii, Pseudomonas aeruginosa, and the Enterobacteriaceae family with different results (Furfaro et al., 2018; Altamirano and Barr, 2019), but no clinical trial using bacteriophages against mycobacteria has been performed. Finally, Brix et al. (2020) summarized different animal models used to study phage therapy, including Caenorhabditis elegans, Drosophila melanogaster, Galleria mellonella, zebrafish, quail, chicken, rabbit, hamster, and mouse.

Phage utilization against Listeria monocytogenes, Salmonella enterica serovar Typhimurium and Escherichia coli have been approved for food industry. These products have been named as Generally Recognized As Safe (GRAS) by the US Food and Drug Administration (FDA), however, no approval is available for standard therapeutic use to date (Sarhan and Azzazy, 2015).

\section{SUCCESSFUL CASE REPORT AGAINST MYCOBACTERIUM ABSCESSUS}

More than 10,000 mycobacteriophages have been isolated to date from different sources, and most of the registered phages infect Mycobacterium smegmatis (The Actinobacteriophage Database, 2020; Table 1). This database shows just three phages infecting $M$. abscessus GD01 (one sequenced) and one phage infecting $M$. abscessus subsp. bolletii F1660. The 17\% of total mycobacteriophages have been sequenced and classified into 29 clusters, 71 sub-clusters, and 10 singletons (Sinha et al., 2020). Phages infecting Mycobacterium genus are tailed with doublestranded DNA and belong to Siphoviridae and Myoviridae families, which harbor long flexible non-contractile tails and contractile tails, respectively (Hatfull, 2018). Mycobacteriophages have been traditionally utilized as tools for diagnosis or genetic manipulation.

Thirteen phages (DS-6A, TM4, D29, T7, P4, PDRPv, BTCU-I, Bo4, SWUI, GR-2I/T, My-327, Ms6, and Bxz2) were recently reviewed as candidates for phage therapy against Mycobacterium 
TABLE 1 | Registered phages infecting Mycobacterium genus.

\begin{tabular}{lcc}
\hline Species (N strains) & Number of phages & Sequenced phages \\
\hline M. abscessus (2) & 3 & 1 \\
M. aichiense (1) & 1 & 1 \\
M. aurum (1) & 4 & 0 \\
M. avium (7) & 9 & 7 \\
M. phlei (2) & 8 & 8 \\
M. smegmatis (2) & 11,388 & 1,931 \\
M. sp. (1) & 1 & 0 \\
M. tuberculosis (1) & 1 & 1 \\
\hline
\end{tabular}

N strains: number of strains (The Actinobacteriophage Database, 2020).

tuberculosis and other mycobacteria (Azimi et al., 2019). Another mycobacteriophage (ZoeJ) related to TM4 has been described as a broad host-range infecting $M$. tuberculosis, M. smegmatis, and Mycobacterium avium (Dedrick et al., 2019a).

The only study published about phage therapy against M. abscessus is the case report published in 2019 of a 15-yearold patient suffering from cystic fibrosis (CF) who had a double lung transplant. The patient had been treated for 8 years against chronic $P$. aeruginosa and $M$. abscessus subsp. massiliense infections. After bilateral lung transplant, immunosuppressive drugs and intravenous antibiotics were delivered. Reddening of surgical wound was noted 1 week after finishing intravenous administration. Mycobacterium abscessus was isolated and intravenous antibacterial antibiotics were restarted (amikacin, imipenem/cilastatin, and tigecycline) together with clofazimine and bedaquiline. Infection evolved and 7 months post-transplant the patient suffered from a disseminated infection due to M. abscessus with skin lesions. At this point, phage therapy was considered as compassionate use. Screening of more than 10,000 M. smegmatis phages against M. abscessus lead to the selection of three bacteriophages (Siphoviridae family). The treatment consisted of a cocktail of the three phages introduced by intravenous route, two of them were genetically engineered to delete repressors and convert temperate phages into lytic phages. Phages were detected in serum 1 day after starting, falling below detection limits 6 days afterward. After starting the phage therapy, no M. abscessus was isolated from serum or sputum. Finally, the patient was discharged home with antibiotics and phage administration and no adverse effects were reported. Improvement of lungs, liver, and skin was achieved after 7 months of intravenous infusions of phages every $12 \mathrm{~h}$ $\left(10^{9} \mathrm{PFU} /\right.$ dose of each phage) and topical administration of phages daily in combination with multiple antibiotics (amikacin, imipenem/cilastatin, tigecycline, bedaquiline, and clofazimine; Dedrick et al., 2019b). This was the first case of phage therapy using genetically modified phages and the first use in patients against a mycobacterial infection.

A recent report about requests for phage therapy at the Center for Innovative Phage Applications and Therapeutics (IPATH) in the United States shows that from June 1, 2018, to April 30, 2020, there have been 90 outcomes of requests for phage therapy against mycobacteria (47 M. abscessus, 23 $M$. avium, seven Mycobacterium chimera, seven $M$. species, two Mycobacterium chelonae, and one M. smegmatis, Mycobacterium xenopi, Mycobacterium bolletii, and Mycobacterium genavense). In a summarizing table, they point that nine lytic phages were found against the isolates of $M$. abscessus and the phage therapy was administered to four patients with a median time from request to administration of 176 days, however, no more information is given about these treatments (Aslam et al., 2020).

There is a lack of preclinical studies about phage therapy against M. abscessus; however, current preclinical models of M. abscessus infections are diverse. A persister assay for M. abscessus has been recently developed (Yam et al., 2020), which could be used to test phage efficacy against persister variants. Notably, Sb-1 phage was able to direct kill $S$. aureus persisters in vitro (Tkhilaishvili et al., 2018). Interestingly, the first mouse model of pulmonary $M$. abscessus infection in an immunocompetent mouse strain has been recently described (Maggioncalda et al., 2020). Traditionally, immunocompromised mice have been used for murine infection models due to the rapid clearance of $M$. abscessus by immunocompetent hosts (Obregón-Henao et al., 2015). CF mouse models also exist and would be desirable to test preliminary phage efficacy in CF patients (Guilbault et al., 2007).

\section{TIPS FOR PHAGE THERAPY AGAINST MYCOBACTERIUM ABSCESSUS}

\section{Environmental Reservoirs}

Phages are ubiquitous in the environment including the human body; nevertheless, every phage will be encountered near its bacterial host. More than 17,000 actinobacteriophages have been described and the genome diversity of the sequenced phages has been studied (Hatfull, 2020). Mycobacterium abscessus is able to survive in adverse environments and is found in water sources, soil, and also in sinks, showerheads, or sewage (Lopeman et al., 2019). Novel lytic mycobacteriophages infecting M. abscessus should be searched in similar scenarios to directly proceed to the phage enrichment with the clinical bacterium, which is responsible of the infection. Due to phage specificity, tailored treatments are necessary to maximize success of phage therapy, although this personalized manufacture undoubtedly will raise the time from request to administration.

\section{Cocktail of Phages to Tackle Bacterial Resistance and Insensitivity}

Phage resistance in bacteria includes mutations that modify or hide phage receptors, destruction of viral DNA using nucleases or restriction enzymes, and via CRISPR-Cas system (Labrie, 2010). Modifications in polysaccharides, teichoic acids, and outer membrane proteins block phage adsorption in Grampositive bacteria. However, phage-resistant variants are often balanced with bacteria-fitness costs usually associated with decreased virulence or higher susceptibility to antibiotics (Mangalea and Duerkop, 2020), although a faster growth-rate derivative has also been described (Kashiwagi and Yomo, 2011). Temperate phages may represent an obstacle for the entry of invasive phages, the analysis of temperate mycobacteriophages 
infecting M. tuberculosis and M. smegmatis exhibited at least five types of defense systems that avoided phage infection (Dedrick et al., 2017), although phages can in turn escape these mechanisms (Hatfull, 2020). Prophages are abundant in bacterial genomes including mycobacteria. A recent study of prophages within clinical NTM concluded that it was more likely to find prophages in rapidly compared to slowly growing bacteria and also suggested that prophages from clinical mycobacteria may contain more virulence genes than environmental mycobacteria (Glickman et al., 2020). Importantly, a genome analysis of $48 \mathrm{M}$. abscessus strains concluded that at least 17 mycobacteriophages have infected M. abscessus during its evolution and the authors located one to eight prophage regions in 47 out of the 48 genomes, from intact prophages to small prophage-like elements (Sassi et al., 2014). A singleparticle electron microscopy reconstruction of a prophage infecting M. abscessus subsp. bolletii was reported (64-kb genome; Sassi et al., 2013). To overcome bacterial resistance and the presence of temperate phages in $M$. abscessus, phage therapy should use cocktails of phages which, in addition, broaden the spectrum of infection.

\section{Antibiotic-Phage Combinations}

The mycobacteriophage SWU1 gp39 has been proposed as an enhancer of isoniazid, erythromycin, norfloxacin, ampicillin, ciprofloxacion, ofloxacin, rifampicin, and vancomycin antibiotics against M. smegmatis via modification of cell wall permeability (Li et al., 2016, 1). Of special interest is the effect of phages on biofilms after producing enzymes that breakdown the extracellular matrix enabling metabolic revival of bacteria and antibiotic arrival (Chakraborty and Kumar, 2019). Interestingly, subcutaneous administration of lysin B (a D29 enzyme) was able to prevent bacteria proliferation in a mouse model of Mycobacterium ulcerans footpad infection (Fraga et al., 2019). These combinations could diminish doses and timing of antibiotics against $M$. abscessus reducing adverse effects and toxicity of long treatments.

\section{Liposomal Administration}

Mycobacteriophages are able to target extracellular bacteria; however, intracellular variants inside macrophages are difficult to access. Encapsulated phages into liposomes are promising for different reasons such as prolonged effect, intracellular delivery, protection against neutralizing antibodies, and preservation from stomach acidic $\mathrm{pH}$ in case of oral delivery (Colom et al., 2015; Malik et al., 2017). TM4 mycobacteriophages encapsulated into giant liposomes were shown to enter eukaryotic cells more efficiently than free phages (Nieth et al., 2015). Interestingly, inhaled liposomal amikacin have been evaluated in a randomized phase II clinical trial for NTM-lung-infected patients and results show improvements in sputum conversion (Olivier et al., 2017). One important issue for phage-therapy efficacy is the accessibility of the pathogen to the phages. For this reason, diverse deliveries should be used depending of the site of infection. In case of pulmonary infection, an inhalation delivery could be administered. A comparative study in the delivery rate of D29 phage against $M$. tuberculosis revealed that vibrating mesh nebulizer was more efficient than soft mist inhaler and jet nebulizer, indicating that vibrating mesh nebulizer would be recommended to deliver large amounts of phage (Carrigy et al., 2017). Intravenous phage administration would be desirable to deliver phages throughout the body in systemic and/or disseminated infections caused by M. abscessus, and in case of skin and soft tissue infections local application of phages, including intramuscular, subcutaneous, or topical administration should be studied.

\section{Preferential Use in Patients Suffering From Cystic Fibrosis}

Persistent infections in CF patients after lung transplant can result in significant morbidity and mortality (Furukawa and Flume, 2018). Mycobacterium abscessus has been detected in $7 \%$ of CF patients in United Kingdom (UK Cystic Fibrosis Registry, 2020). CF patients present increased levels of nuclear factor $\mathrm{kB}(\mathrm{NF} \kappa \mathrm{B})$ with chronic inflammation (Bodas, 2010), and also aberrant trafficking and degradation of Toll-like receptor 4 (TLR4), thereby enhancing inflammatory response (Bruscia et al., 2011). Moreover, rough variants of $M$. abscessus enhance IFN-I and IL- $1 \beta$ production via mitochondrial ROS (Kim et al., 2020). Phages are believed to promote immunomodulating and anti-inflammatory actions, are able to diminish ROS in vitro (Górski et al., 2020) and reduce NFкB activation (Górski et al., 2006). Mice fed with T7 phage showed only a slight increase in IL-17A compared to control mice (Park et al., 2014). Surprisingly, another study reported IFN gamma induction by STA-8505 phage, which infects $S$. aureus (Pincus et al., 2015), indicating that more in vivo studies are needed to clarify this point. Remarkably, phages are more abundant in mucosal surfaces via binding between mucin glycoproteins and Ig-like protein domains of phages protecting the underlying epithelium from bacterial infection (Barr et al., 2013); this characteristic may increase the multiplicity of infection against the target bacteria in lungs of CF patients. Finally, the biofilm destruction by phages could represent an additional advantage to deal with pulmonary infections efficiently in CF patients.

\section{CONCLUDING REMARKS}

Mycobacterium abscessus infections require novel approaches. Phage therapy combined with antibiotics represents a promising alternative against $M$. abscessus due to the expected synergistic effect of phages with antibiotics against planktonic bacteria and biofilms. The studies reviewed here lead to considering some productive future research to answer remaining questions about phage therapy against $M$. abscessus: Can cocktails of phages overcome narrow host range and phage resistance against $M$. abscessus? Or is it only possible as tailor-made, personalized therapy? Which antibiotic-phage combination will be the most effective against M. abscessus? Can antibiotics combined with mycobacteriophages achieve higher effect with lower doses and timing, reducing adverse effects associated to prolonged use? Which route of 
administration will be adequate in each type of M. abscessus infection (pulmonary, systemic and/or disseminated infections, skin and soft-tissue infections)? Can intracellular M. abscessus be eliminated using phages embedded into liposomes? To what extent will phages destroy biofilms in patients with inflammatory lung diseases, such as cystic fibrosis, bronchiectasis, and chronic obstructive pulmonary disease? Specific mouse models of these infections exist. Are limitations of these murine models a big deal? Which type of patients would benefit from a prophylactic and therapeutic phage administration? There are still limitations that should be addressed prior to apply this approach into the clinical practice; however, phage therapy could be currently considered a realistic option under compassionate use.

\section{REFERENCES}

Altamirano, F. L. G., and Barr, J. J. (2019). Phage therapy in the postantibiotic era. Clin. Microbiol. Rev. 32, e00066-e00118. doi: 10.1128/CMR.00066-18

Aslam, S., Lampley, E., Wooten, D., Karris, M., Benson, C., Strathdee, S., et al. (2020). Lessons learned from the first 10 consecutive cases of intravenous bacteriophage therapy to treat multidrug-resistant bacterial infections at a single center in the United States. Open Forum Infect. Dis. 7:ofaa389. doi: 10.1093/ofid/ofaa389

Azimi, T., Mosadegh, M., Nasiri, M. J., Sabour, S., Karimaei, S., and Nasser, A. (2019). Phage therapy as a renewed therapeutic approach to mycobacterial infections: a comprehensive review. Infect. Drug Resist. 12, 2943-2959. doi: 10.2147/IDR.S218638

Barr, J. J., Auro, R., Furlan, M., Whiteson, K. L., Erb, M. L., Pogliano, J., et al. (2013). Bacteriophage adhering to mucus provide a non-host-derived immunity. Proc. Natl. Acad. Sci. U. S. A. 110, 10771-10776. doi: 10.1073/pnas.1305923110

Bento, C. M., Gomes, M. S., and Silva, T. (2020). Looking beyond typical treatments for atypical mycobacteria. Antibiotics 9:18. doi: 10.3390/antibiotics 9010018

Bodas, M. (2010). The NFkB signaling in cystic fibrosis lung disease: pathophysiology and therapeutic potential. Discov. Med. 9, 346-356.

Brix, A., Cafora, M., Aureli, M., and Pistocchi, A. (2020). Animal models to translate phage therapy to human medicine. Int. J. Mol. Sci. 21:3715. doi: 10.3390/ijms21103715

Bruscia, E. M., Zhang, P. -X., Satoh, A., Caputo, C., Medzhitov, R., Shenoy, A., et al. (2011). Abnormal trafficking and degradation of TLR4 underlie the elevated inflammatory response in cystic fibrosis. J. Immunol. 186, 6990-6998. doi: 10.4049/jimmunol.1100396

Carrigy, N. B., Chang, R. Y., Leung, S. S. Y., Harrison, M., Petrova, Z., Pope, W. H., et al. (2017). Anti-tuberculosis bacteriophage D29 delivery with a vibrating mesh nebulizer, jet nebulizer, and soft mist inhaler. Pharm. Res. 34, 2084-2096. doi: 10.1007/s11095-017-2213-4

Catherinot, E., Clarissou, J., Etienne, G., Ripoll, F., Emile, J. -F., Daffé, M., et al. (2007). Hypervirulence of a rough variant of the Mycobacterium abscessus type strain. Infect. Immun. 75, 1055-1058. doi: 10.1128/IAI.00835-06

Chakraborty, P., and Kumar, A. (2019). The extracellular matrix of mycobacterial biofilms: could we shorten the treatment of mycobacterial infections? Microb. Cell 6, 105-122. doi: 10.15698/mic2019.02.667

Chalmers, J. D., Aksamit, T., Carvalho, A. C. C., Rendon, A., and Franco, I. (2018). Non-tuberculous mycobacterial pulmonary infections. Pulmonology 24, 120-131. doi: 10.1016/j.pulmoe.2017.12.005

Colom, J., Cano-Sarabia, M., Otero, J., Cortés, P., Maspoch, D., and Llagostera, M. (2015). Liposome-encapsulated bacteriophages for enhanced oral phage therapy against Salmonella spp. Appl. Environ. Microbiol. 81, 4841-4849. doi: 10.1128/AEM.00812-15

Daley, C. L., Iaccarino, J. M., Lange, C., Cambau, E., Wallace, R. J., Andrejak, C., et al. (2020). Treatment of nontuberculous mycobacterial pulmonary disease: an official ATS/ERS/ESCMID/IDSA clinical practice guideline. Clin. Infect. Dis. 71, 905-913. doi: 10.1093/cid/ciaal125

Dedrick, R. M., Bustamante, C. A. G., Garlena, R. A., Pinches, R. S., Cornely, K., and Hatfull, G. F. (2019a). Mycobacteriophage ZoeJ: a broad host-range

\section{AUTHOR CONTRIBUTIONS}

MG-Q and JE conceived the manuscript. AS-K, MG-Q, and JE critically reviewed the literature. AS-K and MG-Q wrote the manuscript. JE revised the manuscript. All authors contributed to the article and approved the submitted version.

\section{FUNDING}

MG-Q is supported by the Subprograma Miguel Servet from the Ministerio de Ciencia e Innovación of Spain, the Instituto de Salud Carlos III and the European Union, European Social Fund "Investing in your future" (CP19/00104).

close relative of mycobacteriophage TM4. Tuberculosis 115, 14-23. doi: 10.1016/j.tube.2019.01.002

Dedrick, R. M., Guerrero-Bustamante, C. A., Garlena, R. A., Russell, D. A., Ford, K., Harris, K., et al. (2019b). Engineered bacteriophages for treatment of a patient with a disseminated drug resistant Mycobacterium abscessus. Nat. Med. 25, 730-733. doi: 10.1038/s41591-019-0437-z

Dedrick, R. M., Jacobs-Sera, D., Bustamante, C. A. G., Garlena, R. A., Mavrich, T. N., Pope, W. H., et al. (2017). Prophage-mediated defence against viral attack and viral counter-defence. Nat. Microbiol. 2:16251. doi: 10.1038/ nmicrobiol.2016.251

Fraga, A. G., Trigo, G., Murthy, R. K., Akhtar, S., Hebbur, M., Pacheco, A. R., et al. (2019). Antimicrobial activity of mycobacteriophage D29 lysin B during Mycobacterium ulcerans infection. PLoS Negl. Trop. Dis. 13:e0007113. doi: 10.1371/journal.pntd.0007113

Furfaro, L. L., Payne, M. S., and Chang, B. J. (2018). Bacteriophage therapy: clinical trials and regulatory hurdles. Front. Cell. Infect. Microbiol. 8:376. doi: $10.3389 /$ fcimb. 2018.00376

Furukawa, B., and Flume, P. (2018). Nontuberculous mycobacteria in cystic fibrosis. Semin. Respir. Crit. Care Med. 39, 383-391. doi: 10.1055/s-0038-1651495

García-Coca, M., Aguilera-Correa, J. -J., Ibáñez-Apesteguía, A., Rodríguez-Sevilla, G., Romera-García, D., Mahíllo-Fernández, I., et al. (2019). Non-pigmented rapidly growing mycobacteria smooth and rough colony phenotypes pathogenicity evaluated using in vitro and experimental models. Pathog. Dis. 77:ftz051. doi: $10.1093 /$ femspd/ftz051

Glickman, C., Kammlade, S. M., Hasan, N. A., Epperson, L. E., Davidson, R. M., and Strong, M. (2020). Characterization of integrated prophages within diverse species of clinical nontuberculous mycobacteria. Virol. J. 17:124. doi: 10.1186/s12985-020-01394-y

Górski, A., Kniotek, M., Perkowska-Ptasińska, A., Mróz, A., Przerwa, A., Gorczyca, W., et al. (2006). Bacteriophages and transplantation tolerance. Transplant. Proc. 38, 331-333. doi: 10.1016/j.transproceed.2005.12.073

Górski, A., Międzybrodzki, R., Węgrzyn, G., Jończyk-Matysiak, E., Borysowski, J., and Weber-Dąbrowska, B. (2020). Phage therapy: current status and perspectives. Med. Res. Rev. 40, 459-463. doi: 10.1002/med.21593

Guilbault, C., Saeed, Z., Downey, G. P., and Radzioch, D. (2007). Cystic fibrosis mouse models. Am. J. Respir. Cell Mol. Biol. 36, 1-7. doi: 10.1165/rcmb.2006-0184TR

Hatfull, G. F. (2018). Mycobacteriophages. Microbiol. Spectr. 6. doi: 10.1128/ microbiolspec.GPP3-0026-2018

Hatfull, G. F. (2020). Actinobacteriophages: genomics, dynamics, and applications. Annu. Rev. Virol. 7, 37-61. doi: 10.1146/annurev-virology-122019-070009

Haworth, C. S., Banks, J., Capstick, T., Fisher, A. J., Gorsuch, T., Laurenson, I. F., et al. (2017). British thoracic society guideline for the management of nontuberculous mycobacterial pulmonary disease (NTM-PD). BMJ Open Respir. Res. 4:e000242. doi: 10.1136/bmjresp-2017-000242

Johansen, M. D., Herrmann, J. -L., and Kremer, L. (2020). Non-tuberculous mycobacteria and the rise of Mycobacterium abscessus. Nat. Rev. Microbiol. 18, 392-407. doi: 10.1038/s41579-020-0331-1

Kashiwagi, A., and Yomo, T. (2011). Ongoing phenotypic and genomic changes in experimental coevolution of RNA bacteriophage $\mathrm{Q} \beta$ and Escherichia coli. PLoS Genet. 7:e1002188. doi: 10.1371/journal.pgen.1002188 
Kim, B. -R., Kim, B. -J., Kook, Y. -H., and Kim, B. -J. (2020). Mycobacterium abscessus infection leads to enhanced production of type 1 interferon and NLRP3 inflammasome activation in murine macrophages via mitochondrial oxidative stress. PLoS Pathog. 16:e1008294. doi: 10.1371/journal.ppat.1008294

Labrie, S. J. (2010). Bacteriophage resistance mechanisms. Nat. Rev. Microbiol. 8, 317-327. doi: 10.1038/nrmicro2315

Li, Q., Zhou, M., Fan, X., Yan, J., Li, W., and Xie, J. (2016). Mycobacteriophage SWU1 gp39 can potentiate multiple antibiotics against Mycobacterium via altering the cell wall permeability. Sci. Rep. 6:28701. doi: 10.1038/srep28701

Lopeman, R., Harrison, J., Desai, M., and Cox, J. (2019). Mycobacterium abscessus: environmental bacterium turned clinical nightmare. Microorganisms 7:90. doi: $10.3390 /$ microorganisms 7030090

Luepke, K. H., Suda, K. J., Boucher, H., Russo, R. L., Bonney, M. W., Hunt, T. D., et al. (2017). Past, present, and future of antibacterial economics: increasing bacterial resistance, limited antibiotic pipeline, and societal implications. Pharmacotherapy 37, 71-84. doi: 10.1002/phar.1868

Maggioncalda, E. C., Story-Roller, E., Mylius, J., Illei, P., Basaraba, R. J., and Lamichhane, G. (2020). A mouse model of pulmonary Mycobacteroides abscessus infection. Sci. Rep. 10:3690. doi: 10.1038/s41598-020-60452-1

Malik, D. J., Sokolov, I. J., Vinner, G. K., Mancuso, F., Cinquerrui, S., Vladisavljevic, G. T., et al. (2017). Formulation, stabilisation and encapsulation of bacteriophage for phage therapy. Adv. Colloid Interface Sci. 249, 100-133. doi: $10.1016 /$ j.cis.2017.05.014

Mangalea, M. R., and Duerkop, B. A. (2020). Fitness trade-offs resulting from bacteriophage resistance potentiate synergistic antibacterial strategies. Infect. Immun. 88, e00926-e01019. doi: 10.1128/IAI.00926-19

McCallin, S., Sacher, J. C., Zheng, J., and Chan, B. K. (2019). Current state of compassionate phage therapy. Viruses 11:343. doi: 10.3390/v11040343

Meir, M., and Barkan, D. (2020). Alternative and experimental therapies of Mycobacterium abscessus infections. Int. J. Mol. Sci. 21:6793. doi: 10.3390/ ijms 21186793

Muñoz-Egea, M. -C., Carrasco-Antón, N., and Esteban, J. (2020). State-of-theart treatment strategies for nontuberculous mycobacteria infections. Expert Opin. Pharmacother. 21, 969-981. doi: 10.1080/14656566.2020.1740205

Nash, K. A., Brown-Elliott, B. A., and Wallace, R. J. (2009). A novel gene, erm(41), confers inducible macrolide resistance to clinical isolates of Mycobacterium abscessus but is absent from Mycobacterium chelonae. Antimicrob. Agents Chemother. 53, 1367-1376. doi: 10.1128/AAC.01275-08

Nieth, A., Verseux, C., Barnert, S., Süss, R., and Römer, W. (2015). A first step toward liposome-mediated intracellular bacteriophage therapy. Expert Opin. Drug Deliv. 12, 1411-1424. doi: 10.1517/17425247.2015.1043125

Obregón-Henao, A., Arnett, K. A., Henao-Tamayo, M., Massoudi, L., Creissen, E., Andries, K., et al. (2015). Susceptibility of Mycobacterium abscessus to antimycobacterial drugs in preclinical models. Antimicrob. Agents Chemother. 59, 6904-6912. doi: 10.1128/AAC.00459-15

Olivier, K. N., Griffith, D. E., Eagle, G., McGinnis, J. P., Micioni, L., Liu, K., et al. (2017). Randomized trial of liposomal amikacin for inhalation in nontuberculous mycobacterial lung disease. Am. J. Respir. Crit. Care Med. 195, 814-823. doi: $10.1164 / \mathrm{rccm} .201604-0700 \mathrm{OC}$

Park, K., Cha, K. E., and Myung, H. (2014). Observation of inflammatory responses in mice orally fed with bacteriophage T7. J. Appl. Microbiol. 117, 627-633. doi: 10.1111/jam.12565

Patey, O., McCallin, S., Mazure, H., Liddle, M., Smithyman, A., and Dublanchet, A. (2018). Clinical indications and compassionate use of phage therapy: personal experience and literature review with a focus on osteoarticular infections. Viruses 11:18. doi: 10.3390/v11010018

Pincus, N. B., Reckhow, J. D., Saleem, D., Jammeh, M. L., Datta, S. K., and Myles, I. A. (2015). Strain specific phage treatment for Staphylococcus aureus infection is influenced by host immunity and site of infection. PLoS One 10:e0124280. doi: 10.1371/journal.pone.0124280

Reardon, S. (2014). Phage therapy gets revitalized. Nature 510, 15-16. doi: $10.1038 / 510015 a$

Sarhan, W. A., and Azzazy, H. M. (2015). Phage approved in food, why not as a therapeutic? Expert Rev. Anti Infect. Ther. 13, 91-101. doi: 10.1586/ 14787210.2015.990383

Sassi, M., Bebeacua, C., Drancourt, M., and Cambillau, C. (2013). The first structure of a mycobacteriophage, the Mycobacterium abscessus subsp. bolletii phage araucaria. J. Virol. 87, 8099-8109. doi: 10.1128/JVI.01209-13

Sassi, M., Gouret, P., Chabrol, O., Pontarotti, P., and Drancourt, M. (2014). Mycobacteriophage-drived diversification of Mycobacterium abscessus. Biol. Direct 9:19. doi: 10.1186/1745-6150-9-19

Schooley, R. T., Biswas, B., Gill, J. J., Hernandez-Morales, A., Lancaster, J., Lessor, L., et al. (2017). Development and use of personalized bacteriophagebased therapeutic cocktails to treat a patient with a disseminated resistant Acinetobacter baumannii infection. Antimicrob. Agents Chemother. 61, e00954-e01017. doi: 10.1128/AAC.00954-17

Sinha, A., Eniyan, K., Manohar, P., Ramesh, N., and Bajpai, U. (2020). Characterization and genome analysis of B1 sub-cluster mycobacteriophage PDRPxv. Virus Res. 279:197884. doi: 10.1016/j.virusres.2020.197884

The Actinobacteriophage Database (2020). Available at: https://phagesdb.org/ hosts/genera/1/ (Accessed November 13, 2020).

Tkhilaishvili, T., Lombardi, L., Klatt, A. -B., Trampuz, A., and Di Luca, M. (2018). Bacteriophage Sb-1 enhances antibiotic activity against biofilm, degrades exopolysaccharide matrix and targets persisters of Staphylococcus aureus. Int. J. Antimicrob. Agents 52, 842-853. doi: 10.1016/j. ijantimicag.2018.09.006

Tortoli, E., Kohl, T. A., Brown-Elliott, B. A., Trovato, A., Leão, S. C., Garcia, M. J., et al. (2016). Emended description of Mycobacterium abscessus, Mycobacterium abscessus subsp. abscessus and Mycobacterium abscessus subsp. bolletii and designation of Mycobacterium abscessus subsp. massiliense comb. nov. Int. J. Syst. Evol. Microbiol. 66, 4471-4479. doi: 10.1099/ijsem.0.001376

UK Cystic Fibrosis Registry (2020). Available at: https://www.cysticfibrosis.org. uk/the-work-we-do/uk-cf-registry (Accessed September 1, 2020).

Yam, Y. -K., Alvarez, N., Go, M. -L., and Dick, T. (2020). Extreme drug tolerance of Mycobacterium abscessus persisters. Front. Microbiol. 11:359. doi: $10.3389 /$ fmicb. 2020.00359

Conflict of Interest: The authors declare that the research was conducted in the absence of any commercial or financial relationships that could be construed as a potential conflict of interest.

Copyright (c) 2021 Senhaji-Kacha, Esteban and Garcia-Quintanilla. This is an open-access article distributed under the terms of the Creative Commons Attribution License (CC BY). The use, distribution or reproduction in other forums is permitted, provided the original author(s) and the copyright owner(s) are credited and that the original publication in this journal is cited, in accordance with accepted academic practice. No use, distribution or reproduction is permitted which does not comply with these terms. 